\title{
The Hodgkin Huxley Model: Analysis of Dynamic Behavior of the Action Potential in the Giant Squid Axon
}

\author{
S Suresh \\ Department of ECE \\ Raghu Institute of Technology \\ Visakhapatnam \\ India \\ K Gopala Krishna \\ Department of ECE \\ Raghu Institute of Technology \\ Visakhapatnam \\ India
}

\author{
V Bhavani \\ Department of ECE \\ Raghu Institute of Technology \\ Visakhapatnam \\ India \\ Ch Bhaskar Sri Sai \\ Department of ECE \\ Raghu Institute of Technology \\ Visakhapatnam \\ India
}

\begin{abstract}
The main concern of modelling a biological neuron using any electronic circuit to create qualitive models. A nerve cell reacts to a stimulus with a voltage shift or an energy potential gap between the cell and its environment resulting in a spike in voltage. To generate action potential, different methods should be implemented. To improve the propagation of action potential, we use an accurate and efficient method i.e. HodgkinHuxley model.
\end{abstract}

The Hodgkin-Huxley experiment is a quantitative description of the actual movement of the neuronal membrane across ionselective channels, and demonstrated the underpinnings of cell physiology as one of the most revolutionary studies of the 20th century and beyond. Using simple, first-order, ordinary differential equations, Hodgkin and Huxley were able to explain their time behavior using potassium $(\mathrm{K})$ and sodium $(\mathrm{Na})$ streams of intracellular membrane potential and currents. This was done using parameters equipped with a voltage clamp test on the giant axon of the squid. MATLAB simulates the kinetics of ionic currents, effects of alteration of the component currents, and the analysis time step.

Keywords: Hodgkin-Huxley model, cell electrophysiology, biophysics, computational biology.

\section{INTRODUCTION}

We should primarily concentrate on neurons, but it is prudent to begin this analysis at the most fundamental level: the brain. In brain, a cell is an entity that is the smallest structural structure capable of operating independently. Neurons are a particular type of cell. These are trained primarily in sending electric signals to other neurons. There are three separate parts of the neuron that we ought to understand: the cell nucleus, the dendrite, and the axon. The electrical impulse that brings an instruction from the brain to, for example, the hand passes along a chain of neurons [1].
The nerve cell-to-neuron signal transfer happens at the synapse, a fluid flow region between two communicative neurons. The electrical impulse of the presynaptic neurons is translated into a chemical message in the synapse and translated again into an electronic signal in the postsynaptic neurons [2][3].

\subsection{Representations of biological neuronal model:}

These are the most biologically reliable ones. Different model parameters represent those biological components of the neuron. Such models illustrate how the neurons work in depth but are costly to quantify and therefore the simulations appear to be sluggish. We model the capacity of a neuron to combine inputs and fire through a threshold. The HodgkinHuxley model is a neuron point design. Point neuron simulations are concerned mainly with how the neurons treat input voltage to generate or not create an action potential. The Hodgkin-Huxley method is a form of accurate description of the neuron. This model is concerned with how ion motions cause shifts in the voltage of the neurons. Therefore, to understand what this model mimics, the basic knowledge of ion changes is necessary [4].

\section{IONIC MOVEMENT}

The crucial argument that Hodgkin and Huxley were able to show seemed to be that two ion forms, namely sodium (Na) and potassium ( $\mathrm{K}$ ), would adequately clarify the electrical properties of the neurons. Outside the cell the $\mathrm{Na}+$ concentration is higher than inside so these ions are driven by diffusion into the neuron. Often, when sodium ions are drawn into the cell by electrical forces as the cell is 
negatively charged compared to the external cell is shown in figure $1[1]$.

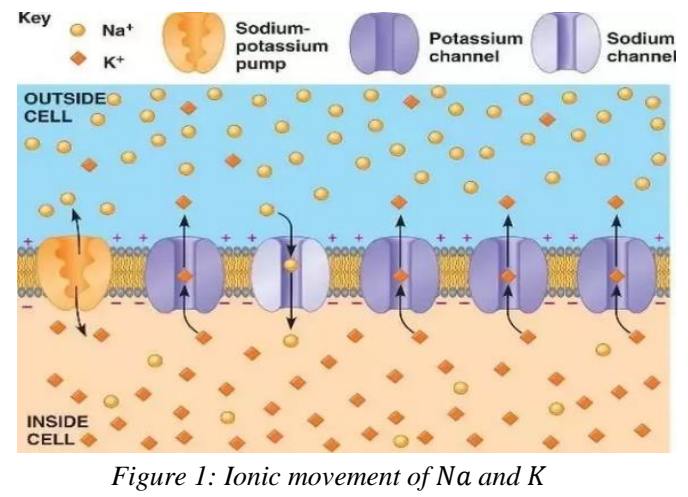

The ions also operate with two forces: diffusion and electrostatic pressure, which influence the ionic flow of the extracellular and intracellular fluid. The diffusion force pushes the ions to disperse the ion uniformly throughout the liquid, with no high or low-concentration regions. Furthermore, as the intracellular fluid contains a high concentration of $K^{+}$, absorption pushes these ions into the extracellular fluid. Electrostatic pressure causes the same charge ions to be repelled, whereas ions with opposite charges are attracted to each other [4].

\subsection{Membrane potential:}

Both cells in the body have electrical impedance or potential differences throughout inside and then outside it. Because the cell membrane differentiates inner surface from the outside, this potential variation is considered as the potential of the membrane. In scientific terms, the $V_{x}$ the cell voltage is described in equation (1)

$\mathrm{V}_{\mathrm{x}}=\mathrm{V}_{\mathrm{i}}-\mathrm{V}_{\mathrm{o}}$

Where $V_{i}$ is the voltage of the inner cell and $V_{o}$ is the voltage of the outer cell. It is going to change during an electrical potential. Capacitance is known as a charge kept divided by the voltage needed to hold the charge [5].

$\mathrm{C}_{\mathrm{x}}=\mathrm{Q} / \mathrm{V}$

This capacitance $C_{x}$ is believed to be continuous, i.e. not time-dependent. Therefore, by taking the time derivative, we can obtain the following equation from the movement of charge across the membrane.

$$
\frac{\mathrm{dQ}}{\mathrm{dt}}=\mathrm{C}_{\mathrm{x}} \frac{\mathrm{dV}}{\mathrm{dt}}
$$

This formula is important to evaluate the membrane potential of the Hodgkin-Huxley model for all the work to be done on this topic [1].

\subsection{Nernst potential:}

The electrical potential produced by the electrochemical balance of the membrane, the equilibrium potential, can be calculated by a simple formula called the Nernst equation.

$V_{x}=\frac{R T}{z F} \ln \left(\frac{[X]_{o}}{[X]_{i}}\right)$
Here $\mathrm{R}$ is the universal gas constant, $\mathrm{T}$ is the absolute temperature of Kelvin, $\mathrm{F}$ is the fixed value of Faraday, and $\mathrm{Z}$ is the valence of the particle. Because three of these parameters are environmental parameters, one often comes across different formulas where these constants have been defined by their importance in the issue under discussion.

\section{HODGKIN HUXLEY MODEL}

The Hodgkin-Huxley model is one among the biological models (Abbott \& Kepler, 1990), utilizes four differential equations to live the potential of the membrane. These four differential equations model the ionic activity of the brain. Hodgkin as well as Huxley carried out experiments on the giant squid axon and found 3 categories of electrons current, i.e. sodium, potassium and leakage current consisting primarily of chlorine ions. The movement of these molecules from the cellular membranes is regulated by different ion-dependent voltage mediums for sodium and potassium ions. Certain channel forms are not explicitly described with the leak current. The semipermeable cellular membrane separates the interior of the cell and acts as a capacitor. From their observations, we were able to derive comprehensive calculations to describe the changes in the ionic current intensity [6].

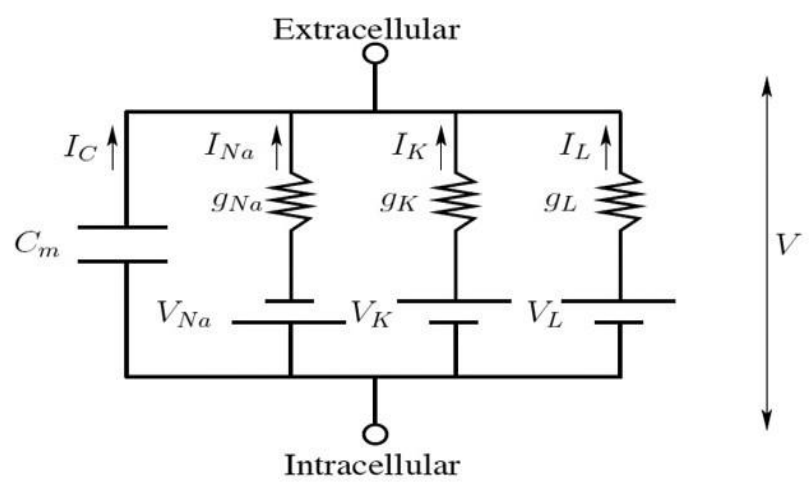

Figure 2: Simplified equivalent circuit for a small section of the giant squid axon

In figure (2), the input current $\mathrm{I}(\mathrm{t})$ is transferred through the cell, either providing significant charge to the capacitance or leakage along the cell membrane mediums. A resistor represents each type of channel. The unspecific channel has a resistance to leakage $R$, a resistance to the sodium channel $R_{N a}$, and a resistance to potassium channel $R_{K}$. The concentration of the ion within the neuron varies from the one in the extracellular material by the intensive transport of the ion across the cellular membrane. Nernst potential is different from every sort of ion. The sodium , potassium and undefined leakage channel voltages are $V_{N a}, V_{k}$ and $V_{L}$ respectively.

The conversation of electrical charge on a membrane piece indicates that the current $I x(\mathrm{t})$ added can be separated into capacitance current $I c$ that affects the condenser $\mathrm{C}$ and $I_{k}$ that passes across ion mediums [6] 
$\mathrm{I}_{\mathrm{x}}(\mathrm{t})=\mathrm{Ic}(\mathrm{t})+\sum_{\mathrm{i}} \mathrm{I}_{\mathrm{i}}(\mathrm{t})$

Where $\sum_{\mathrm{i}} \mathrm{I}_{\mathrm{i}}(\mathrm{t})=\mathrm{I}_{\mathrm{Na}}+\mathrm{I}_{\mathrm{K}}+\mathrm{I}_{\mathrm{L}}$

The theory of electrical circuits states that the amount of voltage shift over the capacitor is equal to the maximum current used to charge it, or

$\operatorname{Ix}(\mathrm{t})=\mathrm{Cx} \frac{\mathrm{dV} x}{\mathrm{dt}}+\mathrm{I}_{\mathrm{n}}$

Where the current among three channels is,

$\mathrm{I}_{\mathrm{n}}=\mathrm{G}_{\mathrm{N}_{\mathrm{a}}} * \mathrm{~V}_{\mathrm{x}}-\mathrm{V}_{\mathrm{Na}}+\mathrm{G}_{\mathrm{k}} * \mathrm{~V}_{\mathrm{x}}-\mathrm{V}_{\mathrm{k}}+\mathrm{G}_{\mathrm{L}} * \mathrm{~V}_{\mathrm{x}}-\mathrm{V}_{\mathrm{L}}$

Whereas its leakage channel is defined by a voltage dependent conductance $G_{L}=1 / R$. Because $V_{x}$ is the overall potential around the membrane and $V_{L}$ is the reversal potential across leaky channel, the voltage at the leaky channel is $V_{x}-V_{L}$.

By applying ohm's rule at leaky current

$\mathrm{I}_{\mathrm{L}}=\mathrm{G}_{\mathrm{L}}\left(\mathrm{V}_{\mathrm{x}}-\mathrm{V}_{\mathrm{L}}\right)$

The time-dependent membrane current $I x$ is

$\operatorname{Ix}(\mathrm{t})=\mathrm{Cx} \frac{\mathrm{dV} \mathrm{x}}{\mathrm{dt}}+\mathrm{G}_{\mathrm{Na}_{\mathrm{a}}} * \mathrm{~V}_{\mathrm{x}}-\mathrm{V}_{\mathrm{Na}}+\mathrm{G}_{\mathrm{k}} * \mathrm{~V}_{\mathrm{x}}-\mathrm{V}_{\mathrm{K}}+\mathrm{G}_{\mathrm{L}} *$

$\mathrm{V}_{\mathrm{x}}-\mathrm{V}_{\mathrm{L}}$

Hodgkin-Huxley equation:

$\mathrm{Ix}(\mathrm{t})=\mathrm{Cx} \frac{\mathrm{dV} \mathrm{x}}{\mathrm{dt}}+\overline{\mathrm{G}_{\mathrm{Na}}} \mathrm{m}^{3} \mathrm{~h} * \mathrm{~V}_{\mathrm{x}}-\mathrm{V}_{\mathrm{Na}}+\overline{\mathrm{G}_{\mathrm{k}}} \mathrm{n}^{4} * \mathrm{~V}_{\mathrm{x}}-\mathrm{V}_{\mathrm{K}}+$

$\overline{\mathrm{G}}_{\mathrm{L}} * \mathrm{~V}_{\mathrm{x}}-\mathrm{V}_{\mathrm{L}}$

Where $V_{N a}, V_{k}, V_{L}$ are reversal potentials

When this mediums are free, it transfer currents among a greater conductance $\overline{G_{N a}}, \overline{G_{k}}, \bar{G}_{L}$. Even then, a few streams are being blocked. $\mathrm{m}, \mathrm{n}$ and $\mathrm{h}$ are gating aspects that are created to design the possibility that a channel will be opened at a given time. The cumulative constants of $\mathrm{m}$ and h handles the $N_{a}$ channels while K gates are handled by $\mathrm{n}$ constant is shown in equation (8).

The efficient conductivity of the sodium channels is defined as $1 / R_{N a}=\overline{G_{N a}} m^{3} h$ where $\mathrm{m}$ specifies the initiation (opening) of the medium and $\mathrm{h}$ the termination(closing/blocking) of the medium. Potassium conductivity is $1 / R_{K}=\overline{G_{k}} n^{4}$ while $\mathrm{n}$ represents the initiation of the channel [6].

The change in the gating variables is based on the following differential equations:

$\frac{\mathrm{dn}}{\mathrm{dt}}=\alpha_{\mathrm{n}} * \mathrm{~V}_{\mathrm{x}} * 1-\mathrm{n}-\beta_{\mathrm{n}} * \mathrm{~V}_{\mathrm{x}} * \mathrm{n}$

$\frac{\mathrm{dm}}{\mathrm{dt}}=\alpha_{\mathrm{m}} * \mathrm{~V}_{\mathrm{x}} * 1-\mathrm{m}-\beta_{\mathrm{m}} * \mathrm{~V}_{\mathrm{x}} * \mathrm{~m}$

$\frac{d h}{d t}=\alpha_{h} * V_{x} * 1-h-\beta_{n} * V_{x} * h$

\section{VOLTAGE-CLAMP USED BY HODGKIN AND HUXLEY}

In many of the studies conducted by Hodgkin and Huxley, an electrode was injected into the squid axon to keep the membrane at a fixed voltage. Figure 3 shows the calculated current flow from the axon of a giant squid through a specified region of neural membrane to calculate how ions move through the neuron membrane during an action potential [8]. The voltage-clamp enabled the ionic currents to be registered directly, flowing through the giant axon's axonal membrane without any subsequent membrane potential adjustments. A simple voltage clamp can iteratively calculate the membrane potential, and then adjust the membrane potential (voltage) by applying the required current to the target value. Its "clamps" the cell membrane at the ideal voltage constant, enabling the clamp to monitor what currents are transmitted. The voltage-clamp eliminated capacitance problems and created an isopotential membrane [8].

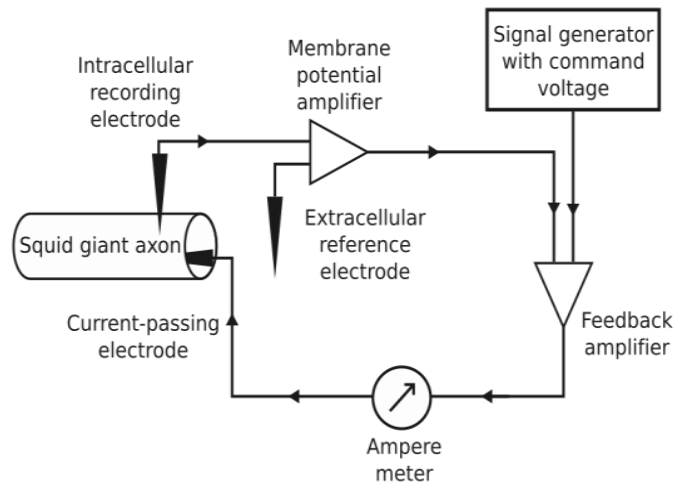

Figure 3: Voltage clamp of a squid axon

\section{HODGKIN-HUXLEY MODEL USING EULER'S METHOD}

The computational method of Hodgkin and Huxley was one convenient for manual computation by means of the tools at that time. The task of determining the value at a given time is an integral part of the differential equation. The difficulty with this is that the four differential equations are interrelated and therefore it is difficult to calculate the integral. Then, an approximation of the equation is used, often by the Euler method. In Euler's process, there is a parameter that is the time step dt, which, based on the values selected, which make it impossible for the model to function or make it take too long to operate. A general first-order differential equation of the form.

$\frac{\mathrm{dy}(\mathrm{t})}{\mathrm{dt}}=\mathrm{f} * \mathrm{y}(\mathrm{t})$

Therefore, in the computer analysis of Hodgkin-Huxley calculations, one needs to be careful when picking a discreet time stage duration. Sensitive protection is to seek small variations in the chosen value for $\Delta t[7]$.

Applying Euler's method to the membrane voltage equation, 
$V_{x}(t+\Delta t)=\left(1-\frac{\Delta t}{\tau_{i}}\right) V_{x}(t)+\frac{\Delta t}{\tau_{N a}} E_{N a}+\frac{\Delta t}{\tau_{k}} E_{k}+\frac{\Delta t}{\tau_{l k}} E_{l k}$

\section{SIMULATION OF HODGKIN-HUXLEY}

Each section displays the action potential along with the related plots of the time for gating parameters. Such plots were obtained by computer simulations using the forward Euler method technique with differential equations. There is a time stage of $1 \mu \mathrm{s}$. The $\mathrm{HH}$ model simulated its action potential with an applied stimulus from $\mathrm{t}=0 \mathrm{~ms}$ to $2 \mathrm{~ms}$. The $G_{N a}$ and $G_{k}$ conductances can change with time, since these correspond to the $\mathrm{Na}$ and $\mathrm{K}$ channel opening and closing shown in figure 4 [5].

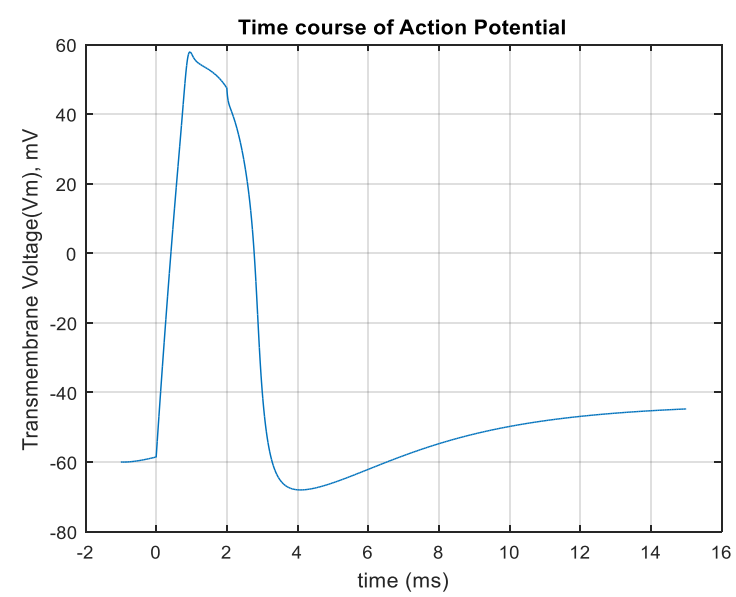

Figure 4: The action potential generated by an exterior pulse width.

The velocity constants $\alpha$ and $\beta$ can be determined using matlab technique as the function of the cell voltage $\mathrm{V}$ and the temperature T. Parameters V (in $\mathrm{mV}$ ) and temperature $\mathrm{T}$ (in Celsius) are transferred to the operations. Figure 5 shows the equilibrium constants at $\mathrm{T}=6.3^{\circ} \mathrm{C}[8]$.
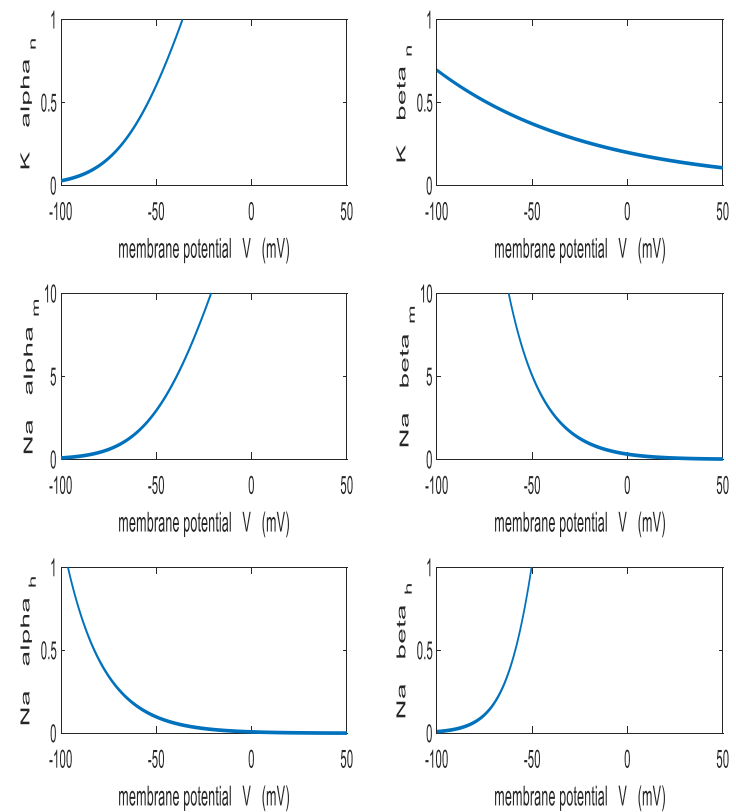

Figure 5: Figures of the equilibrium constants $\alpha$ and $\beta$ at $T=6.3{ }^{\circ} \mathrm{C}$.
Figure 6 shows the reaction of $m, h$, and $n$ to the step-in potential[4]. In figure 7, it shows how the difference in the gate factors, the conductances and the current densities of the voltage-clamp added to the axon. Because the step variable is increased, the variables of gates $(n, m, h)$ then the conductances $\left(g_{N a}\right.$ and $g_{K}$, ) then the current densities are calculated for each time step.

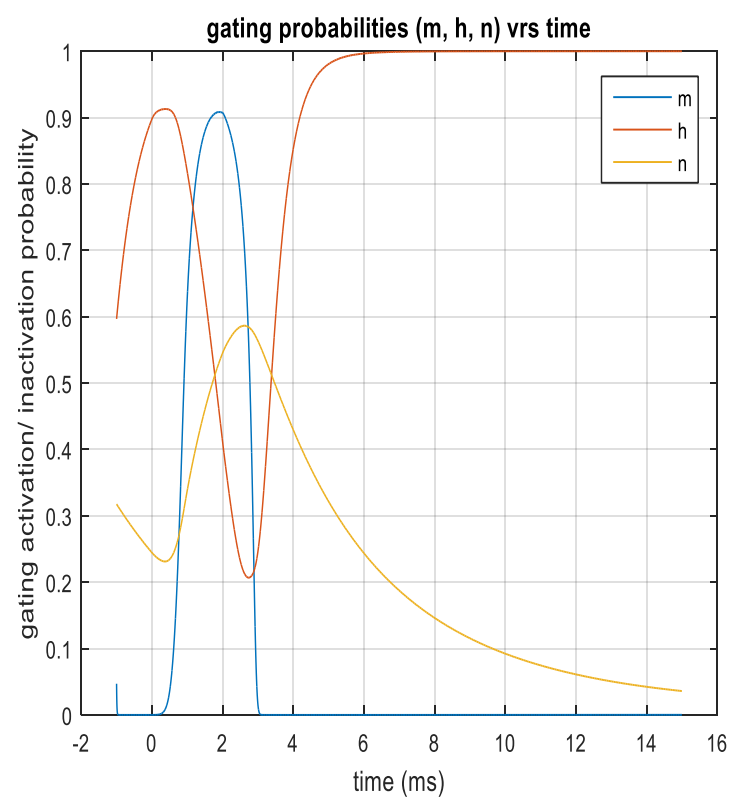

Figure 6: Reaction of the opening and closing parameters $m, h$ and $n$ to the step-in voltage.
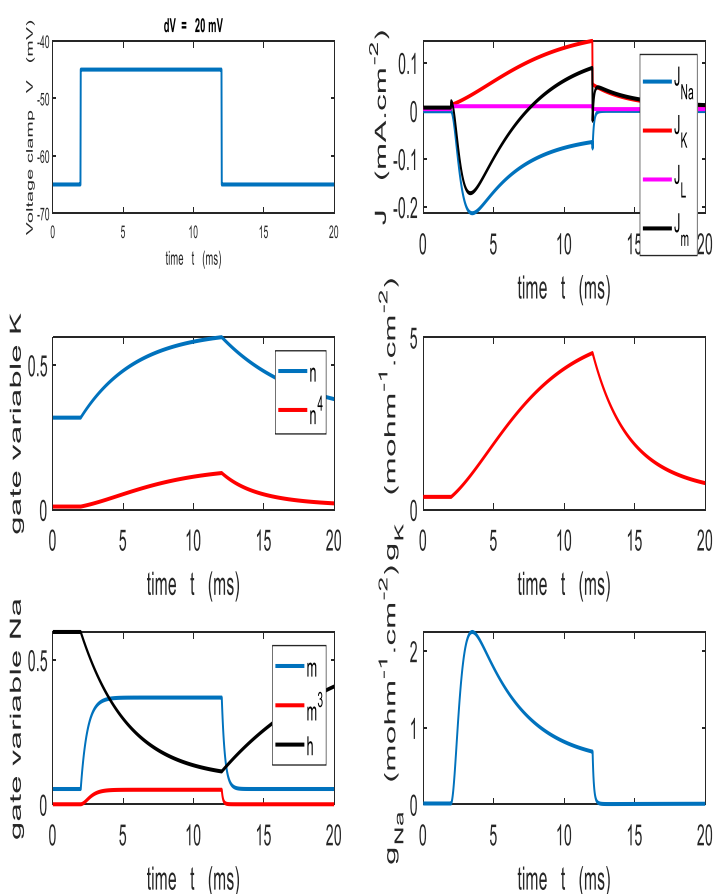

Figure 7: Modification of the gate quantities, conductances and current densities for the voltage clamp added to the axon 
Figure 8 shows solutions in a voltage clamp squid axon at $6.3^{\circ} \mathrm{C}$ for measuring the action potential produced by a 0.010 $\mathrm{mA} . \mathrm{cm}^{-2}$.
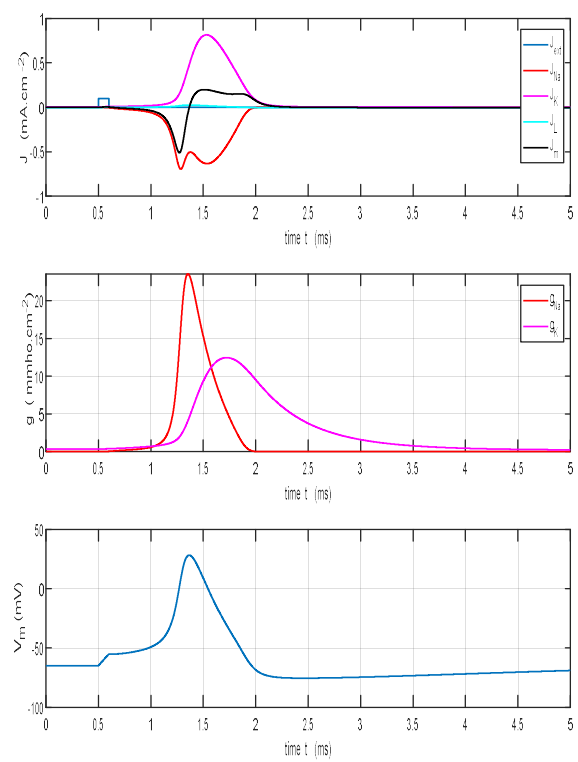

Figure 8: Figures for measuring the action potential produced in a voltage clamp squid axon at $6.3^{\circ} \mathrm{C}$ by an external stimulus of $0.010 \mathrm{~mA} . \mathrm{cm}^{-2}$.

Figure 9 displays the outer control plots that are phase inputs for current densities (uniform current instillation). On time $t$ $=5.0 \mathrm{~ms}$ the triggers are turned on. Unless the phase size is less than $0.007 \mathrm{~mA} . \mathrm{cm}-2$, there is no scope for generation[8].
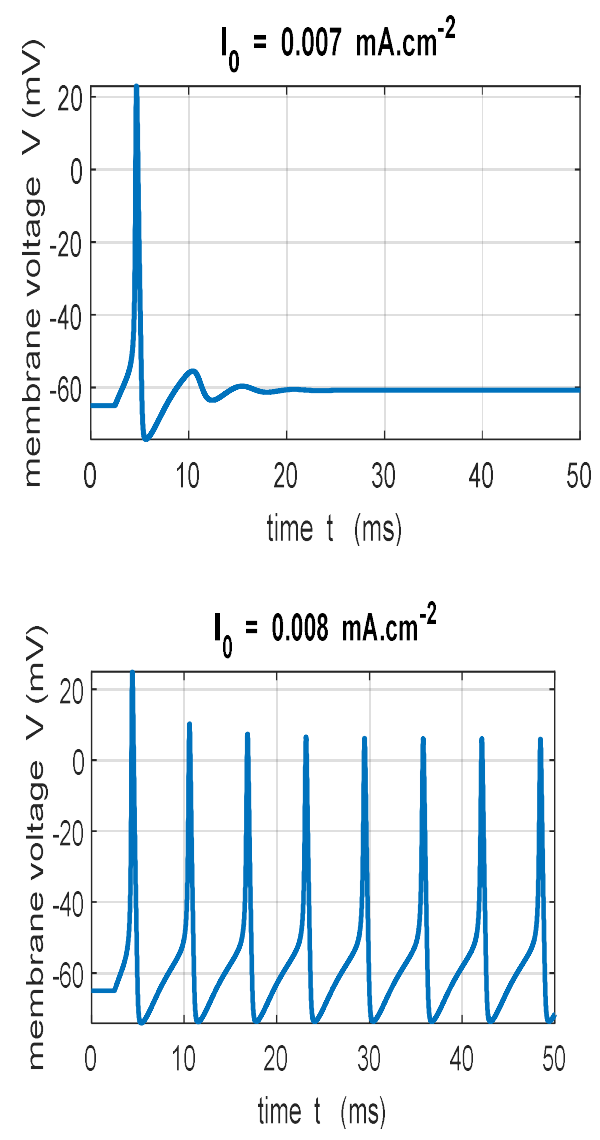
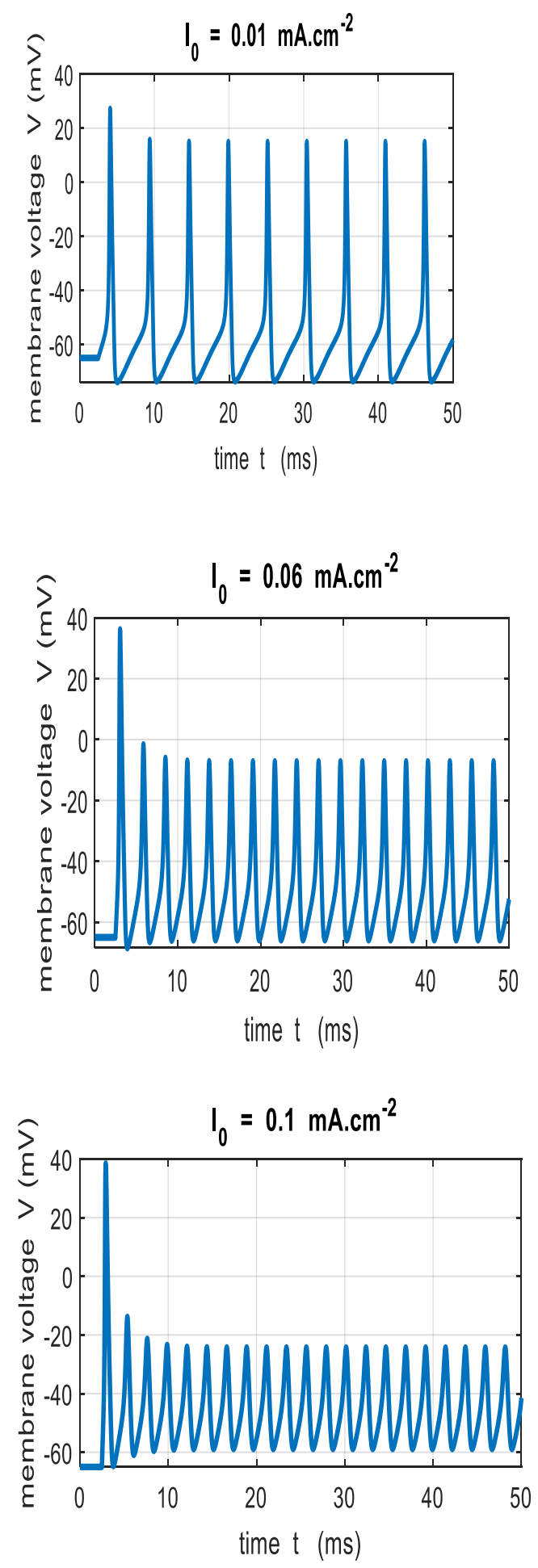

Figure 9: External stimuli are phase triggers for densities of the current

\section{CONCLUSION}

Generating and propagating the action potential deals with the interaction of the conductance of sodium and 
potassium-voltage dependence. In the original Hodgkin and Huxley formulations, important assumptions were made about how the voltage can affect the conductance. The key goal is to provide a comprehensive physical account of all circumstances and kinetic variations between the channel molecule states. The model suggested is beneficial for study of neuronal coding involving neuronal networks.

\section{REFERENCES}

[1] Thierry Mondeel, "Modelling Neuronal Excitation: The HodgkinHuxley Model", July 13, (2012).

[2] Kandel ER, Schwartz JH, Jessell TM, Siegelbaum SA, Hudspeth AJ. Principles of Neural Science. New York, NY: McGraw-Hill; (2013).

[3] Purves D, Augustine GJ, Fitzpatrick D, et al. Neuroscience. Sunderland, MA: Sinauer Associates, Inc; (2012).

[4] Melissa G. Johnson and Sylvain Chartier, "Spike neural models: The Hodgkin-Huxley model", (2017), vol.13.

[5] G.B. Ermentrout and D.H. Terman, Mathematical Foundations of Neuroscience, Interdisciplinary Applied Mathematics 35, DOI 10.1007/978-0-387-87708-2 1, c Springer Science + Business Media, LLC (2010).

[6] Wulfram Gerstner, Werner M. Kistler, Richard Naud and Liam Paninski, "Neuronal Dynamics", published with Cambridge University Press in July (2014).

[7] Richard B. Wells, "Biological Signal Processing", chapter 3, (2003).

[8] Ian Cooper, "Hodgkin-Huxley Model: Membrane Current". 